\title{
Circular RNA SIPA1L1 regulates osteoblastic differentiation of stem cells from apical papilla via miR-204-5p/ALPL pathway
}

\author{
Yuzhi $\mathrm{Li}^{1,2+}$, Minxia Bian ${ }^{1,2+}$, Zhou Zhou ${ }^{1,2+}$, Xiao $\mathrm{Wu}^{1,2}$, Xingyun Ge ${ }^{1}$, Tong Xiao ${ }^{1,2}$ and Jinhua Yu ${ }^{1,2^{*}}$ (D)
}

\begin{abstract}
Background: Osteogenesis is a complex biological process which requires the coordination of multiple molecular mechanisms. This research aimed to explore the biological role and underlying regulatory mechanism of circSIPA1L1 during the osteogenic differentiation of stem cells from apical papilla (SCAPs).
\end{abstract}

Methods: EdU retention assay, flow cytometry assay, and CCK-8 assay were used to evaluate the proliferation capacity of SCAPs. Western blot assay, alkaline phosphatase (ALP), and alizarin red staining (ARS) were conducted to investigate the biological roles of circSIPA1L1 and miR-204-5p. Fluorescence in situ hybridization was applied for circSIPA1L1 localization. Dual-luciferase reporter assay was performed to prove the interaction of circSIPA1L1 and miR-204-5p.

Results: CircSIPA1L1 had no significant effect on the proliferative capacity of SCAPs. CircSIPA1L1 promotes osteogenic differentiation of SCAPs by serving as a miRNA sponge for miR-204-5p. Either knockdown of circSIPA1L1 or overexpression of miR-204-5p significantly suppresses osteogenic differentiation of SCAPs.

Conclusions: CircSIPA1L1 upregulates ALPL through targeting miR-204-5p and promotes the osteogenic differentiation of SCAPs.

Keywords: circRNAs, miR-204-5p, ALPL, SCAPs, Differentiation

\section{Background}

Cell-based tissue regeneration gives the field of regenerative medicine a boost. Mesenchymal stem cells (MSCs) present their superiority in individual treatment of tissue engineering based on their self-renew and multi-differentiation ability. They can differentiate into a variety of cell types such as osteocytes, chondrocytes, and adipocytes $[1,2]$. As promising mesenchymal stem cells, SCAPs are isolated from human immature impacted permanent teeth, their essential roles in the

\footnotetext{
*Correspondence: yujinhua@njmu.edu.cn

${ }^{\dagger}$ Yuzhi Li, Minxia Bian and Zhou Zhou contributed equally to this work. 'Key Laboratory of Oral Diseases of Jiangsu Province and Stomatological Institute of Nanjing Medical University, 136 Hanzhong Road, Nanjing 210029, Jiangsu, China

${ }^{2}$ Endodontic Department, School of Stomatology, Nanjing Medical University, Nanjing, Jiangsu, China
}

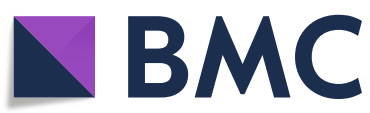

(c) The Author(s). 2020 Open Access This article is licensed under a Creative Commons Attribution 4.0 International License, which permits use, sharing, adaptation, distribution and reproduction in any medium or format, as long as you give appropriate credit to the original author(s) and the source, provide a link to the Creative Commons licence, and indicate if changes were made. The images or other third party material in this article are included in the article's Creative Commons licence, unless indicated otherwise in a credit line to the material. If material is not included in the article's Creative Commons licence and your intended use is not permitted by statutory regulation or exceeds the permitted use, you will need to obtain permission directly from the copyright holder. To view a copy of this licence, visit http://creativecommons.org/licenses/by/4.0/. The Creative Commons Public Domain Dedication waiver (http://creativecommons.org/publicdomain/zero/1.0/) applies to the data made available in this article, unless otherwise stated in a credit line to the data. dental pulp and dentin formation, alveolar bone regeneration, and tooth root growth have been extensively studied [3]. Increasing evidences have demonstrated that SCAPs could exhibit advantages of proliferative capacity and differentiation ability such as adipogenic, osteogenic, and neurogenic $[4,5]$. Besides, many previous studies have indicated the bone tissue formation and regeneration capacities of SCAPs [6-8].

Circular RNAs (circRNAs) are a class of naturally occurring non-coding RNAs, which are abundant in the eukaryotic transcriptome and involve in regulating physiological processes $[2,9,10]$. Compared with traditional linear RNAs, circRNA molecules have a typical closed-loop structure, which is resistant to digestion with RNA exonuclease. The circular structure maintains its stability and strengthens its miRNA/protein-binding 
capacity [11, 12]. As a new molecular biomarker, circRNAs have brought fresh expectations for the diagnosis, treatment, and prognosis of clinical diseases [13]. However, circRNAs were considered to be byproducts of abnormal splicing with little functionality when they were first discovered 25 years ago. With the evolution of RNA deep sequencing technology and bioinformatics analyses, more and more circRNAs have been discovered in eukaryotes, and they present tissue-specific expression patterns $[14,15]$. There is increasing evidence that circRNAs are indispensable in neuronal function, cancer progression, cell proliferation, and differentiation [1618]. Generally, circRNAs act as microRNA' (miRNA) sponges to binding with miRNAs and modulating mRNAs' function. This regulatory process also called competitive endogenous RNAs (ceRNAs) mechanism. Recently, certain kinds of circRNAs have been verified to be involved in many cell biological processes, including the regulation of cell osteogenic differentiation via the circRNA-miRNAs-mRNA axis [19, 20].

MiRNAs are non-coding, single-stranded RNAs which regulate gene expressions through a post-transcriptional manner, resulting in translation inhibition or mRNA degradation [21]. As a key cell fate regulator, miRNAs are involved in critically important physiological processes of stem cells, such as cell proliferation and differentiation [1, 22, 23]. MiR-204-5p acts as a tumor suppressor to regulate the growth and metastasis of breast cancer and has antitumor effects on melanoma cells $[24,25]$. Besides, studies have shown that miR-204$5 p$ has the function of adipogenic differentiation [26]. In terms of osteogenic differentiation, a study proved that LncRNA TUG1 sponge miR-204-5p in calcific aortic valve disease then upregulated post-transcriptional expression of Runx2, leading to the promotion of osteogenic differentiation [27]. Moreover, Chen et al. demonstrated that miR-204-5p may be a potential upstream regulator factor in aging osteoblasts as it is significantly upregulated in an osteoporotic fractured bone. On the other hand, multiple indispensable genes related to osteoblasts differentiation and bone mineralization have been revealed. Among these genes, ALPL (alkaline phosphatase) which is recognized as an early expression gene associated with osteogenesis has attracted our attention [28].. The alkaline phosphatase (ALP) protein it encodes has been widely used as a diagnostic indicator to assess the grade osteoporosis [29]. Researches have shown that ALPL inhibits the sensitivity of bone aging by specifically regulating the differentiation and senescence of MSCs [30].

Our previous RNA-sequencing data revealed that different expression profiles of circRNAs, mRNAs, and miRNAs undergoing osteoblast differentiation of SCAPs. The competitive circRNA-miRNA-mRNA networks predicted by miRanda, RNAhybrid, and Target Scan software containing potential targeting relationships were comprehensively integrated. Among them, we obtained a regulatory axis constructed by circSIPA1L1, miR-204-5p, and ALPL [31]. We detected the expression of circSIPA1L1 during osteoblast differentiation of SCAPs. The results showed that the level of circSIPA1L1 is gradually increasing, which indicated that circSIPA1L1 may exert important functions in the osteoblast differentiation of SCAP. Importantly, in the present study, circSIPA1L1 has been confirmed to promote osteogenesis of dental pulp stem cells [32]. However, the function of circSIPA1L1 in the osteogenic differentiation of SCAPs has not been definitively elucidated yet. In this research, we found that circSIPA1L1 had a negative correlation with miR-204-5p and could promote osteogenic differentiation of SCAPs by upregulating the miR-204-5p target gene ALPL.

\section{Materials and methods \\ Tissue collection and cell culture}

Impacted human third molars were acquired from younger patients (17-20 years old) who need a tooth extraction for orthodontic reasons after signed informed consent. This research was supported by the Ethical Committee of the Stomatological School of Nanjing Medical University. The tissue was carefully isolated and clipped from the apical area of the immature root then digested with a mixture of $4 \mathrm{mg} / \mathrm{mL}$ trypsin (Gibco, Life Technologies, MA, USA) and $3 \mathrm{mg} / \mathrm{mL}$ type I collagenase (Gibco). Subsequently, SCAPs were cultured and identified as we previously reported methods [33]. The 3-5 passages of SCAPs were used in subsequent experiments.

\section{Adipogenic differentiation}

SCAPs were planted into $6 \mathrm{~cm}$ culture dishes and cultured with an adipogenic inducing solution (Cyagen Biosciences Inc.) when the cell density reached $60-70 \%$. Cells were cultured at $37^{\circ} \mathrm{C}$ in $5 \% \mathrm{CO}_{2}$ incubator, and the adipogenic differentiation medium was replaced regularly. After adipogenic induction for 28 days, cells were fixed with $4 \%$ paraformaldehyde (PFA) for $30 \mathrm{~min}$ and Oil Red $\mathrm{O}$ staining was carried out at room temperature.

\section{Chondrogenic differentiation}

SCAPs $\left(4 \times 10^{5}\right.$ cells $)$ were collected and cultured in 15 $\mathrm{ml}$ centrifuge tubes with chondrogenic induction medium (Cyagen Biosciences Inc.) in a $37^{\circ} \mathrm{C}, 5 \% \mathrm{CO}_{2}$ incubator, and loosen the cap of the tube slightly. After 3-5 days, cell pellets were transferred to 24-well plates to culture for 20 days. Cell pellets were stained with Alcian Blue after fixation and frozen sections $(5-\mu \mathrm{m}$ thickness). 


\section{Plasmid construction and cell transfection}

SCAPs was transfected with miR-204-5p mimics (mimics, $50 \mathrm{nM}$ ) and mimics negative control (NC, 50 $\mathrm{nM}$ ), miR-204-5p inhibitor (inhibitor, $100 \mathrm{nM}$ ), and inhibitor negative control (iNC, $100 \mathrm{nM}$ ) at $30-50 \%$ confluence with riboFECT $T^{\text {max }}$ CP kit (Ribobio, Guangzhou, China). Similarly, the knockdown of circSIPA1L1 and negative control groups (si-SIPA1L1, si-NC, $100 \mathrm{nM}$ ) were also transfected in the same reagent. However, the circSIPA1L1 overexpression (sh-SIPA1L1, sh-NC) transfection was performed using lipofectamine 2000 (Invitrogen, USA). SCAPs were cultured with osteogenic induced medium (OM). Moreover, the circSIPA1L1 and ALPL luciferase reporter plasmids were constructed by site-directed mutagenesis and transfected with lipofectamine 2000 under serum-free medium in $293 \mathrm{~T}$ cells for 4-6 $\mathrm{h}$ then exchanged basic culture medium.

\section{Reverse transcription polymerase chain reaction (RT-PCR) analysis}

SCAPs were transfected with several oligonucleotides mentioned above separately in an osteogenic induction medium. Then, total RNA was collected from SCAPs in each group by using TRIzol reagent (Invitrogen). The mRNA gets converted to cDNA by reverse transcription with a Prime Script RT Master Mix kit (TaKaRa, Dalian, China). For the analysis of the levels of miRNA, extracted RNA was specifically reverse transcribed using the transcription kit (Ribobio, Guangzhou, China). GAPDH was used as a housekeeping gene for mRNAs and circRNAs and $U 6$ was chosen as an endogenous control for miRNAs, and the data were calculated by $2^{-\Delta \Delta \mathrm{CT}}$ method as previously reported [34]. The primer sequences of the evaluated genes are listed in Table 1.

\section{Western blot analysis}

Three days after transfection, SCAPs were harvested and lysed in RIPA buffer after washing twice by PBS. The

Table 1 Sense and antisense primers for real-time reverse transcription polymerase chain reaction

\begin{tabular}{lll}
\hline Genes & Primers & Sequences (5'-3') \\
\hline ALPL & Forward & ACCTGAGTGCAGAGTGA \\
& Reverse & CTTCCTCCTTGTTGGTT \\
RUNX2 & Forward & TCTTAGAACAAATTCTGCCCTT \\
& Reverse & TGCTTTGGTCTTGAAATCACA \\
OSX & Forward & CCTCCTCAGCTCACCTTCTC \\
& Reverse & GTTGGAGCCCAAATAGAAA \\
CirCSIPA1L1 & Forward & AAACTGGATGAACAAGGGAGAA \\
& Reverse & TGCTTCACTTAAACAGAGGGCTT \\
GAPDH & Forward & GAAGGTGAAGGTCGGAGTC \\
& Reverse & GAGATGGTGATGGGATTTC \\
\hline
\end{tabular}

equivalent amount of proteins was added to $10 \%$ SDS-PAGE and transferred to PVDF membranes after separation. Then, $5 \%$ milk was used to block these membranes. Subsequently, the PVDF membranes were incubated with primary antibody: ALP (ab95462, Abcam), RUNX2 (ab76956, Abcam), OSX (ab22552, Abcam), and GAPDH (AP0060, Bioworld), each was diluted at 1:1000. The membranes were incubated for $1 \mathrm{~h}$ with secondary antibodies after washing with TBST for $30 \mathrm{~min}$. Western Blotting Imaging System was used to detected immunoreactive bands.

\section{Immunofluorescence staining}

After transfection and induction for 3 days, SCAPs were fixed with $4 \%$ PAF in $10 \mathrm{~mm}^{2}$ glass coverslips. Cells were permeabilized with Triton X-100 solution (Beijing, China) for $12 \mathrm{~min}$ and then blocked with goat serum at $37^{\circ} \mathrm{C}$ for $1.5 \mathrm{~h}$. After incubation with ALP and RUNX2 primary antibodies, they were treated with a mixture of secondary antibody with fluorochrome and phalloidin for $1.5 \mathrm{~h}$ in a dark at room temperature. DAPI (Beyotime, China) was applied to stained nuclei. Subsequently, ALP and RUNX2 were observed under the fluorescence microscope (Leica, Germany). ImageJ software was used for quantification.

\section{Alkaline phosphatase (ALP) staining and activity analysis}

The experiment was performed after osteogenic induction for 5 days of transfected SCAPs. ALP staining kit (Beyotime, China) is used to qualitatively detect the expression of ALP as described previously [35]. ALP activity was examined with an ALP activity assay kit (Jiancheng, Nanjing, China). The absorbance was detected in the microplate reader at $520 \mathrm{~nm}$. ALP activity was normalized to the total protein level.

\section{Alizarin red staining (ARS) and cetylpyridinium chloride (CPC) analysis}

Mineralized nodule formation of transfected SCAPs in osteogenic-induced medium was visualized by ARS staining after osteogenic induction for 14 days. Following a fixation with $4 \%$ PAF and a wash with distilled water, SCAPs were dyed with $0.1 \%$ ARS (Sigma-Aldrich) for 30 min. To quantitatively evaluate mineralization nodules, $10 \%$ CPC (Sigma-Aldrich) was used to dissolve the nodules and the absorbance was detected at $560 \mathrm{~nm}$.

\section{Flow cytometry}

Trypsin without EDTA (Beyotime, Haimen, China) was used to collect the transfected cells when they reached $80 \%$. After fixation with $75 \%$ pre-cooled ethanol at $4{ }^{\circ} \mathrm{C}$ overnight, the cell cycle phases (G0/G1, S, and G2/M) were analyzed using FACS flow cytometer after gently washing twice in $0.01 \%$ phosphate-buffered saline (PBS). The proliferative index $(\mathrm{PI}=\mathrm{G} 2 \mathrm{M}+\mathrm{S})$ was used to analyze results. Flow cytometry also used to identify 
SCAPs through cell surface makers. The transfected SCAPs were collected by trypsin and washed with PBS. The samples with different primary antibodies (CD29, CD34, CD45, CD73, CD90) were incubated for $15 \mathrm{~min}$ under dark conditions. Stained cells were analyzed after being rinsed twice with PBS.

\section{Cell proliferation assay}

After SCAPs were transfected with si-SIPA1L1 or shSIPA1L1 and the corresponding negative control, cells were resuspended and reseeded on 96 well plates $(2 \times$ $10^{3}$ cells/well). Equal amount $\alpha$-MEM containing $10 \%$ cell counting kit (CCK)-8 reagent was added into per well at days $0,1,3,5,7,9$, respectively, and incubated for $2 \mathrm{~h}$ under the same environmental conditions. The absorbance was measured at $450 \mathrm{~nm}$ with a microplate reader. The experiment was repeated in triplicate.

Cell-Light $^{\text {tix }}$ EdU Apollo 567 In Vitro Imaging Kit (Ribobio) was used for EdU assay. The transfected SCAP was seeded in a 24-well plate. Cells were fixed after $5 \mathrm{~h}$ incubation with reagent $\mathrm{A}$. Before the cells were treated with $1 \times$ Apollo ${ }^{\circ}$ reaction mixture for $30 \mathrm{~min}$, they were decolorized with $0.5 \%$ Triton X-100. Then, Hoechst 33342 was used to the stained cell nucleus for $30 \mathrm{~min}$. Following a wash with PBS, dyeing results were visualized using a fluorescence microscope. ImageJ software was used cell count to calculate the cell proliferation rate.

\section{Nucleocytoplasmic separation}

The experiment was carried out using the PARIS ${ }^{\mathrm{Tm}}$ Kit as the manufacturer's instructions. $10^{7}$ fresh cells were trypsinized and centrifuged at low speed. PBS was used to rinsed the cell pellet and then was discarded. The cells were resuspended in $400 \mu \mathrm{l}$ of Cell Fractionation Buffer and incubated for $10 \mathrm{~min}$. All experiment was conducted on ice all the time. Then, these samples were centrifuged for $1 \mathrm{~min}$ at $500 \times g$ to obtain nuclear pellet and cytoplasmic supernatant. Afterwards, the nuclear pellet was lysed with $400 \mu \mathrm{l}$ of pre-cold Cell Disruption Buffer after carefully sucking out cytoplasmic fraction. Next, equal quantities of $2 \times$ Lysis/Binding Solution were added to nuclear and cytoplasmic samples respectively at room temp and immediately mix thoroughly by inverting the tube several times or pipetting gently. The sample mixture was centrifuge for $1 \mathrm{~min}$ to pass the filter after mixing gently with anhydrous ethanol. Subsequently, the Wash Solution 1 and Wash Solution 2/3 were applied to complete the washing steps according to the instructions. Finally, the RNA was dissolved with Elution Solution preheated to $95-100^{\circ} \mathrm{C}$. The Nuclear RNA and cytoplasmic RNA were collected for qRT-PCR analysis.

\section{RNA fluorescence in situ hybridization (FISH)}

To detect circSIPA1L1 localization in cells, SCAPs were cultured on slides in 24-well plates. Then, cells were treated with pre-cold $0.5 \%$ Triton X-100 for $5 \mathrm{~min}$ at $4{ }^{\circ} \mathrm{C}$ after fixation with $4 \%$ paraformaldehyde. $200 \mathrm{uL}$ prehybridization was added into each hole to block at $37^{\circ} \mathrm{C}$ for $30 \mathrm{~min}$. At the same time, the hybridization buffer was preheated at $37^{\circ} \mathrm{C}$. Then, the FISH probe mixture was added to the hybridization buffer. Subsequently, the prehybridization was discarded, and SCAPs were incubated in the hybridization buffer containing FISH probe overnight in dark at $37^{\circ} \mathrm{C}$ according to the instructions. Under the condition of avoiding light, cells were sequentially washed with hybridization buffer at $42^{\circ} \mathrm{C}$ as the manufacturer's instructions. Then, DAPI was re-dyed for $10 \mathrm{~min}$ in dark. After washed three times with PBS, the excessive liquid was removed. The images were captured using the LSM 710 confocal microscope (Leica, Germany).

\section{Luciferase reporter assay}

Before plasmid transfection, HEK-293 T cells were seeded into 24-well plates $\left(1 \times 10^{5}\right.$ cell/well $)$ MiR-204-5p and negative control were transfected into HEK-293 T cells respectively on the second day. After $24 \mathrm{~h}$ of incubation, $100 \mathrm{ng}$ circSIPA1L1 or ALPL wild-type reporter plasmid and 20 ng Renilla luciferase (RL) reporter plasmid was cotransfected into HEK-293 T cells when the cell density came up to $80 \%$. The mutation of circSIPA1L1 or ALPL reporter plasmid was used as a control. Dual-Luciferase Reporter Assay System (Promega, Madison, WI) was used to detect the luciferase activity after $48 \mathrm{~h}$.

\section{Statistical analysis}

Based on all experiments conducted independently at least three times, the date was expressed as mean \pm SD and analyzed using SPSS software and GraphPad Prism 5. We analyzed the statistically significant differences using Student's $t$ test and one-way analysis of variance. Image-Pro Plus 5.0 software was employed to measure the grayscale analyses [36]. In figures, NS $=$ not significant, $" P<0.05$ or lower was considered statistically significant.

\section{Results}

Identification of SCAPs and detection of the expression of circSIPA1L1 and miR-204-5p in SCAPs during osteoblast differentiation

Primary SCAPs grew out from tissue after 3 days, as shown in Fig. 1a. Flow cytometry analysis showed that SCAPs positively expressed MSCs markers (CD29, CD90, and CD73), while negatively expressed the hematopoietic markers such as CD34 and CD45 (Fig. 1d). Moreover, Oil Red O, Alizarin red, and Alcian Blue 
A

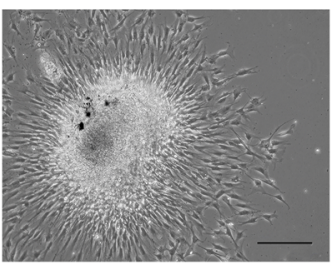

C

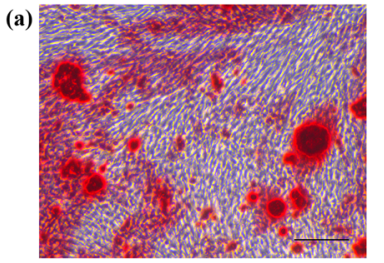

B

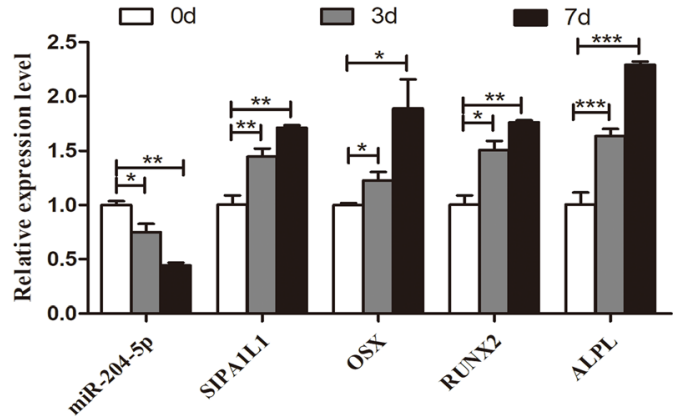

(b)

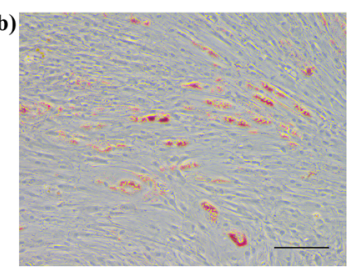

(c)

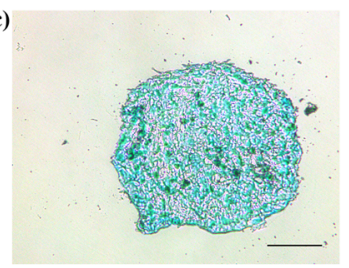

D
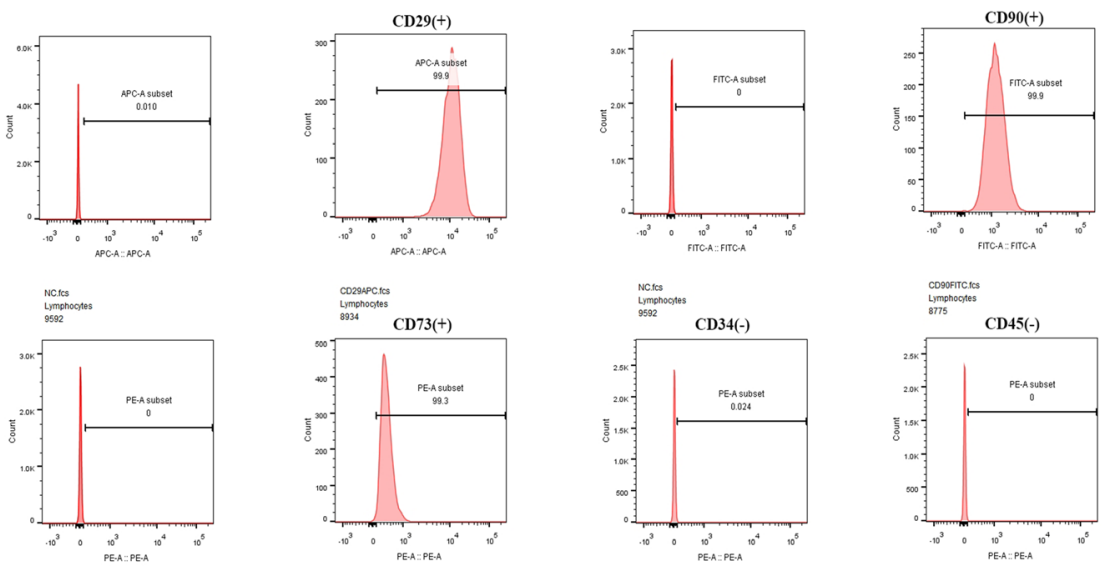

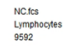

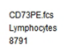

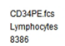

Couspetes
Lmmpoostes
3275

Fig. 1 Identification of SCAPs and expression of circSIPA1L1 and miR-204-5p in SCAPs during osteoblast differentiation. a Morphology of primary SCAPs. $\mathbf{b}$ Relative gene expression of circSIPA1L1, miR-204-5p, and osteoblastic markers of ALPL, RUNX2, and OSX were detected by qRT-PCR at days 0,3 , and 7, respectively. c Tri-lineage differentiation of SCAPs was performed in vitro. a Alizarin red S staining of cells cultured for 14 days in osteogenic induction medium. $\mathbf{b}$ Oil red $\mathrm{O}$ staining of cells cultured for 28 days in adipogenic induction medium. $\mathbf{c}$ Alcian blue staining of cells cultured for 25 days in chondrogenic induction medium. (Scale bar $=200 \mu \mathrm{m}$.) d Flow cytometry demonstrated that SCAPs presented high expressions of CD29, CD90, and CD73, but low expressed CD34 and CD45. ${ }^{*} P<0.05$, ${ }^{*} P<0.01$, and ${ }^{* *} P<0.001$

staining further proved the multi-differentiation ability of SCAPs (Fig. 1c). To investigate the potential role of circSIPA1L1 in the osteogenic differentiation of SCAPs, RNA samples were collected when SCAPs were cultured in osteogenic induction medium 0,3 , and 7 days later. qRT-PCR results demonstrated that the mRNA expressions of $A L P L, R U N X 2$, and OSX were increased obviously, indicating osteogenic induction of SCAPs was successful. Interestingly, the expression of circSIPA1L1 was significantly upregulated under osteogenic induction of SCAPs, while miR-204-5p exhibited the opposite trend $(P<0.05$ or $P<0.01$, Fig. $1 b)$.

\section{CircSIPA1L1 has no effect on SCAPs proliferation}

To investigate whether circSIPA1L1 can exert direct effects on the cell proliferation of SCAPs, flow cytometry assay, CCK- 8 assay, and EdU retention assay were performed. SCAPs transfection was conducted to upregulate or downregulate the expression of circSIPA1L1. The transfection efficacy was obvious by qRT-PCR analysis $(P<0.01$, Fig. $3 \mathrm{~b})$. As the CCK- 8 assay curves showed, $100 \mathrm{nM}$ circSIPA1L1 did not affect cell proliferation (Fig. 2a). In addition, EdU retention assay showed the same results (Fig. 2c, d). Flow cytometry analysis results further confirmed that no apparent difference in the 

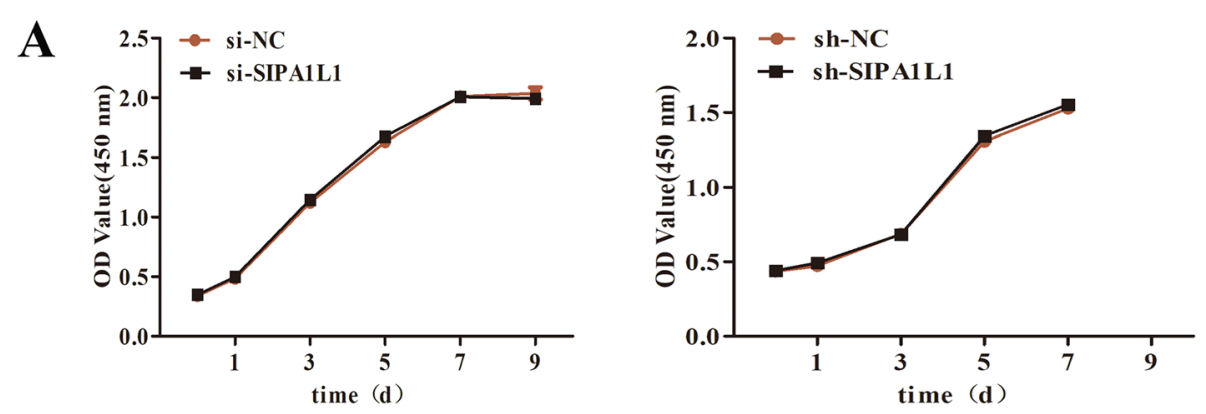

B
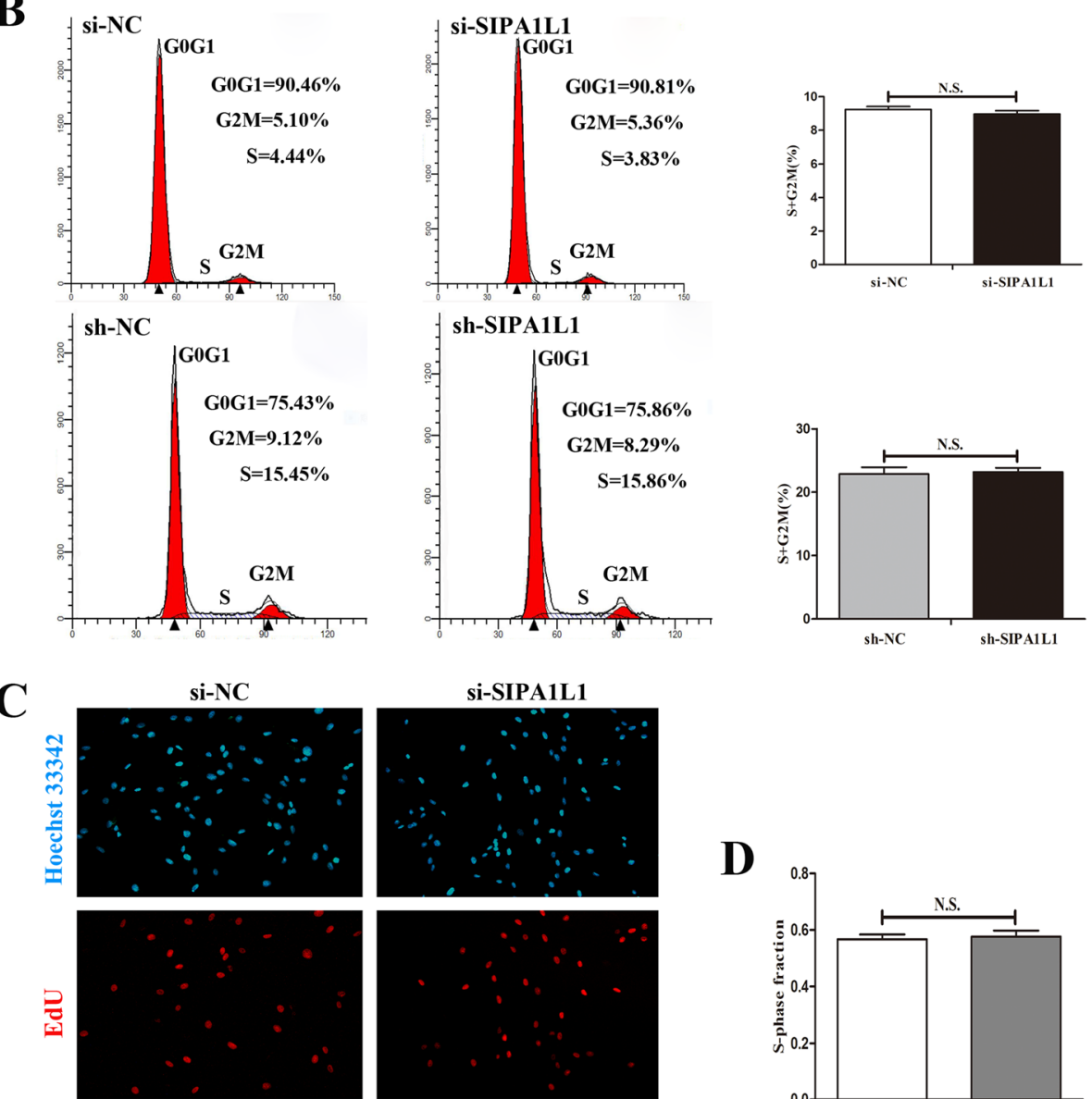

D
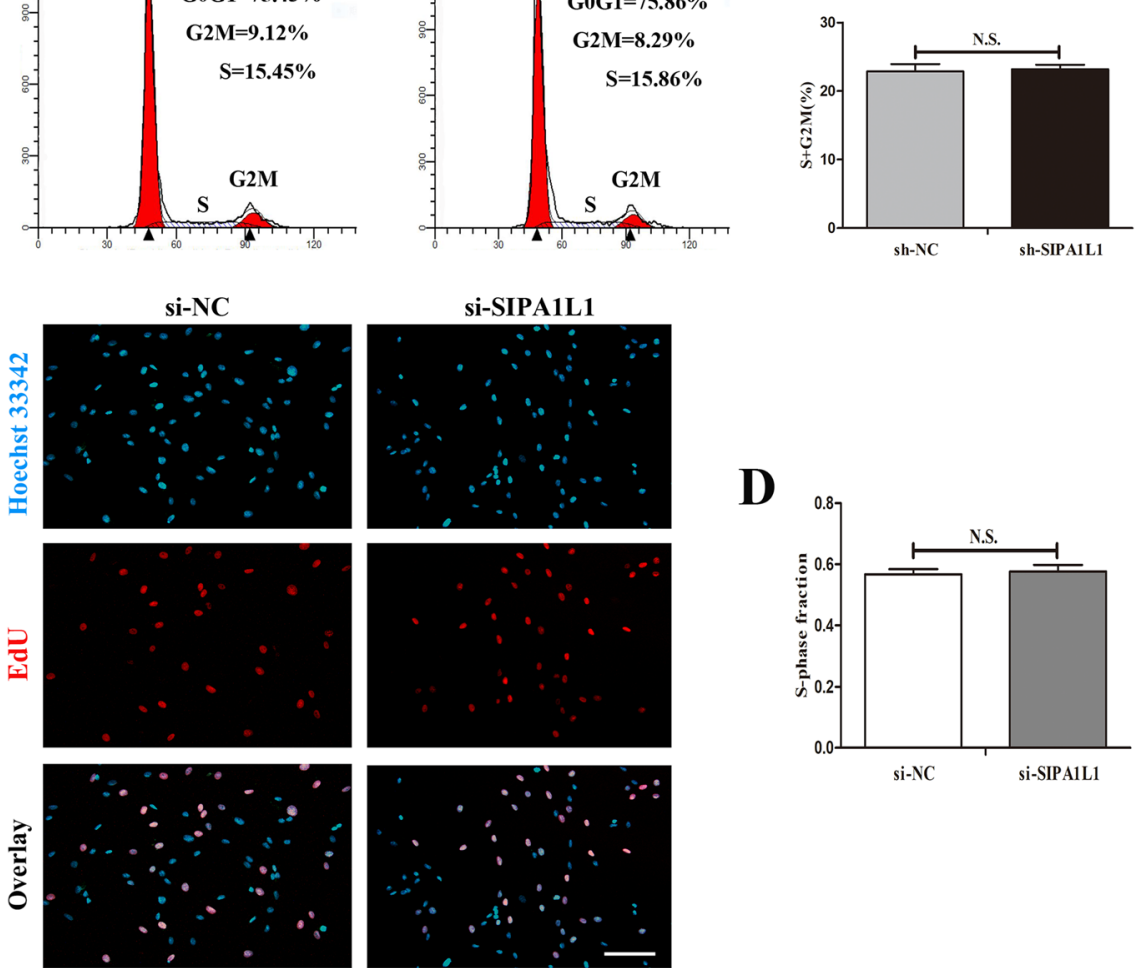

Fig. 2 CircSIPA1L1 no effect on cell proliferation of SCAPs. a CCK-8 assay showed no significant difference in cell proliferation when circSIPA1L1 was overexpression or knockdown. b-d Flow cytometry and EdU assay demonstrated that circSIPA1L1 had no significant difference in cell proliferation of SCAPs. Scale Bar $=200 \mu$ m; N.S., $P>0.05$ 
proliferative index between the circSIPA1L1 group and negative control group $(P>0.05$, Fig. $2 \mathrm{~b})$.

CircSIPA1L1 promotes osteogenic differentiation of SCAPs To assess the involvement of circSIPA1L1 in the regulation of osteogenic differentiation of SCAPs, transfection was conducted in an osteogenic medium to knockdown or overexpression the circSIPA1L1 $(P<0.01$, Fig. 3b). Western blot results showed that expression of ALP, RUNX2, and OSX in si-SIPA1L1 was also markedly downregulated than those in the negative control group, but circSIPA1L1 overexpression obtained the opposite effects $(P<0.05$ or $P<0.01$, Fig. 3a). Meanwhile, the results of qRT-PCR indicated that the mRNA expressions of

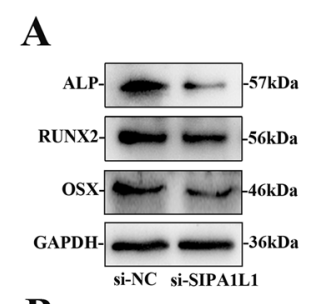

B

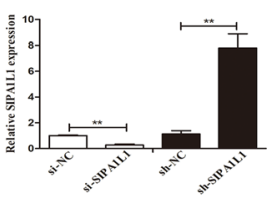

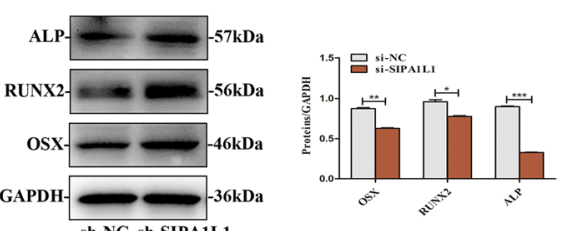

sh-NC sh-SIPA1L1

C

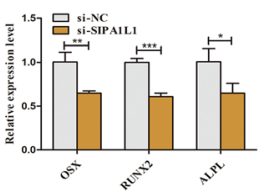

OM 5 days
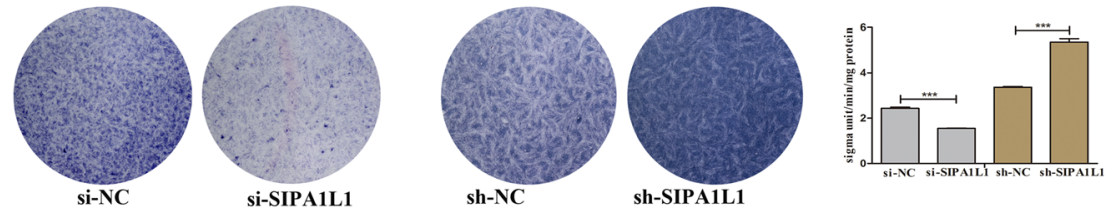

$\mathbf{E}$

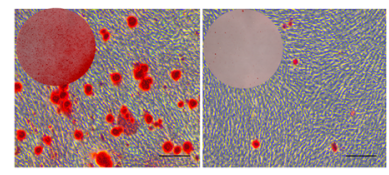

si-NC

Si-SIPA1L1
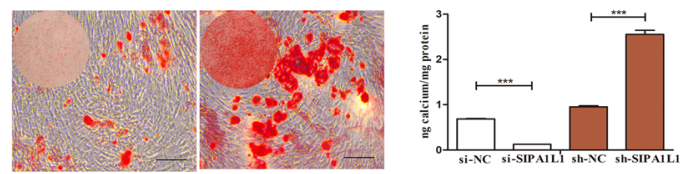

sh-NC

sh-SIPA1L1

F
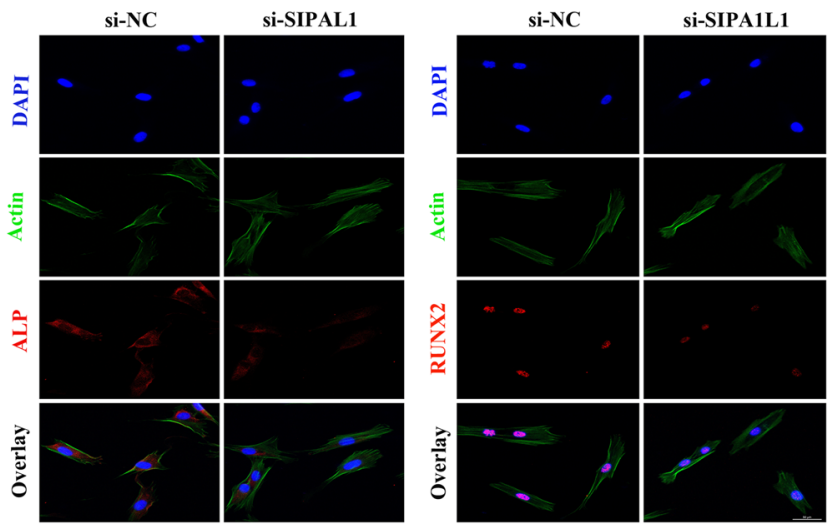

Fig. 3 CircRNASIPA1L1 promotes osteogenic differentiation of SCAPS. a The protein levels of ALP, RUNX2, and OSX in the sh-SIPA1L1 group and siSIPA1L1 group as detected by Western blotting. GAPDH served as an internal control. Histograms showed the quantification of band intensities. $\mathbf{b}$ Transfection efficacy of circSIPA1L1. c Relative mRNA expressions of ALPL, RUNX2, and OSX measured by qRT-PCR. GAPDH was used for normalization. d Images of alkaline phosphatase (ALP) staining in the different groups after being cultured in osteogenic medium (OM) for 5 days. Histograms show ALP activity by spectrophotometry. e Alizarin Red S staining and CPC assay were conducted to investigate the mineralization of SCAPs at day 14 (scale bar $=200 \mu \mathrm{m})$. f ALP and RUNX2 in SCAPs transfected with si-SIPA1L1 or NC were observed by immunofluorescence staining. Scale bar = 50 $\mu$ m. Quantification was done by ImageJ. ${ }^{*} P<0.05$, ${ }^{* *} P<0.01$, and ${ }^{* *} P<0.001$ 
$A L P L, R U N X 2$, and OSX were downregulated by knockdown of circSIPA1L1, whereas a significant increase was observed in overexpression of circSIPA1L1 group $(P<$ 0.05 or $P<0.01$, Fig. $3 \mathrm{c})$. The results of ALP staining and ALP activity detection both presented obviously decline in the si-circSIPA1L1group compared with the si-NC group after induction for 5 days while overexpression
circSIPA1L1 increased ALP activity (Fig. 3d). Besides, after 14 days of osteogenic induction, the intensity of ARS staining and matrix mineralization was distinctly decreased in the si-circSIPA1L1 group, whereas it was notably increased in circSIPA1L1 overexpression group. Moreover, CPC results also showed that the calcium concentration in circSIPA1L1 knockdown group was much

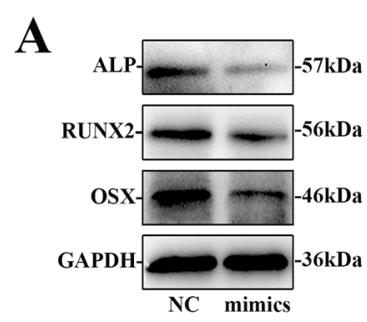

D

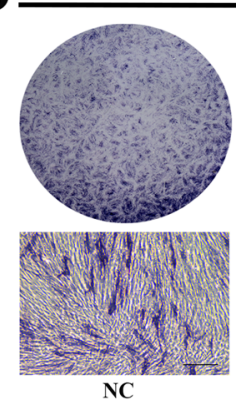

OM 5 days

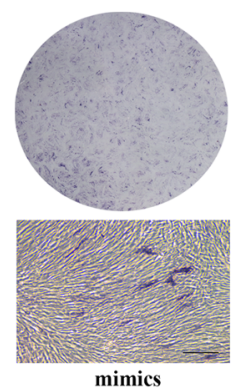

$\mathbf{G}$

$\mathbf{F}$

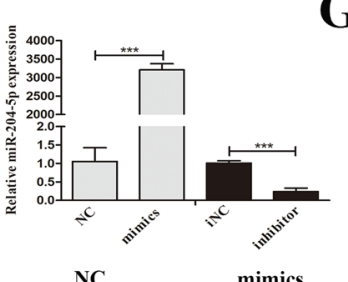

I

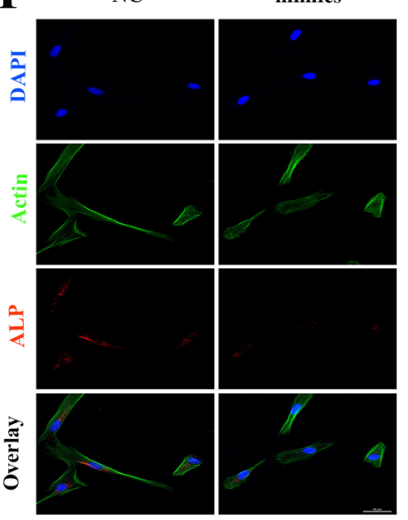

C

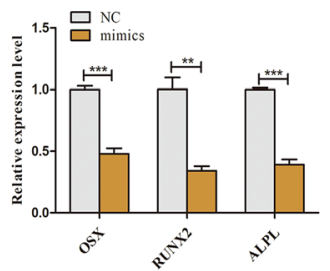

E
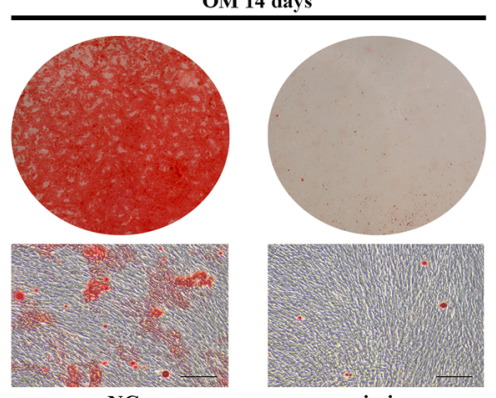

NC

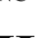

$\mathbf{H}$
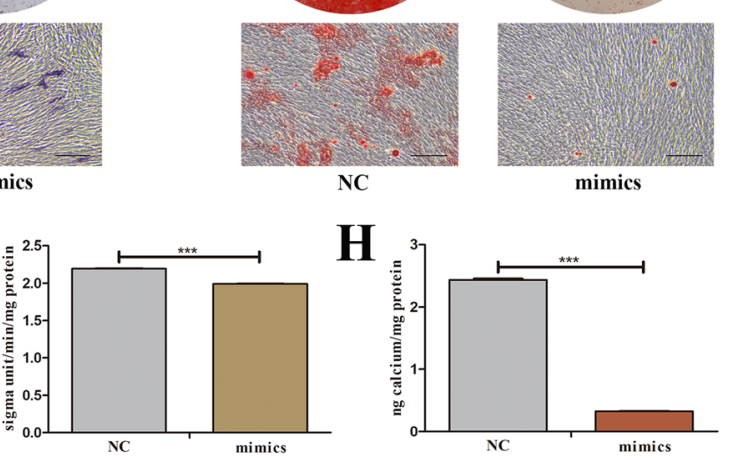

mimics

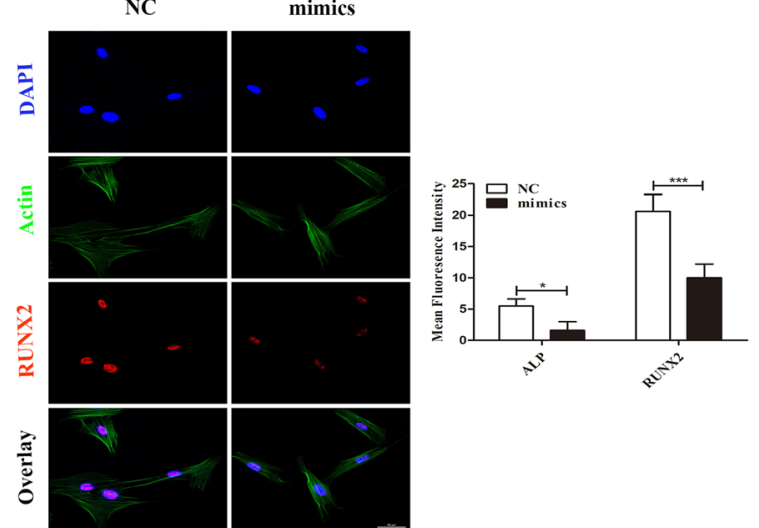

Fig. 4 Overexpression of miR-204-5p suppresses osteogenic differentiation of SCAPs. a Western blot assays were performed to analyze the protein expression levels of ALP, RUNX2, and OSX in the miR-204-5p group than mimics NC group. GAPDH was the internal control. b Grayscale analyses. $\mathbf{c}$ qRT-PCR showed lower levels of ALPL, RUNX2, and OSX in the mimics group. $\mathbf{d}, \mathbf{g}$ Images of ALP staining in the miR-204-5p group. SCAPs were cultured in OM for 5 days. Histograms showed ALP activity by spectrophotometry (bar $=200 \mu \mathrm{m}) \mathbf{e}, \mathbf{h}$ After cell culture in OM for 14 days, Alizarin Red $\mathrm{S}$ staining, and CPC assay showed that miR-204-5p mimics suppresses mineralization of SCAPs (scale bar $=200 \mu \mathrm{m}$ ). f Transfection efficacy of miR-204$5 p$ mimics and inhibitor. $\mathbf{i}$ Immunofluorescence staining showed negative expressions of ALP and RUNX2 in the miR-204-5p mimics group (scale bar $=50 \mu \mathrm{m}$. Quantification was done by ImageJ. ${ }^{*} P<0.05,{ }^{* *} P<0.01,{ }^{* * *} P<0.001$ 
lower than the control group, while it was elevated in circSIPA1L1 overexpression group (Fig. 3e). In addition, the results of the immunofluorescence assay further confirmed that the levels of ALP and RUNX2 were downregulated in the si-SIPA1L1 group (Fig. 3f). Taken together, these results demonstrated that circSIPA1L1 has a positive effect on osteogenic differentiation of SCAPs.

\section{Overexpression of miR-204-5p inhibits osteogenic differentiation of SCAPs}

To explore the functional role of miR-204-5p in the regulation of osteoblast differentiation in SCAPs,
miR-204-5p mimics was used to increased miR-204$5 p$ expression in SCAPs. The results of qRT-PCR revealed that miR-204-5p was obviously increased in the miR-204-5p mimics group and reduced approximately $80 \%$ in the miR-204-5p inhibitor group compared with the respective NC group $(P<0.01$, Fig. $4 \mathrm{f})$. Compared with the NC group, the expression levels of osteogenic differentiation marks (ALP/ALPL, RUNX2/RUNX2, and OSX/OSX) were significantly decreased after additional transfection with miR-204-5p mimics $(P<0.01$, Fig. $4 \mathrm{a}-\mathrm{c})$. In addition, miR-204-5p overexpression decreased ALP activity and reduced
A

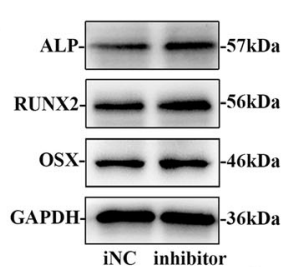

D

OM 5 days

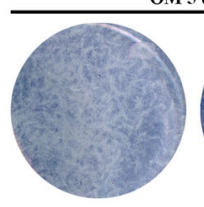

iNC

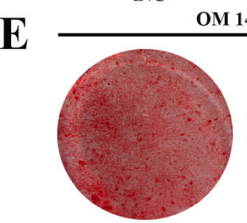

iNC

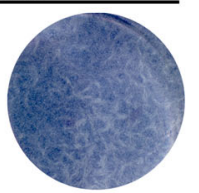

inhibitor

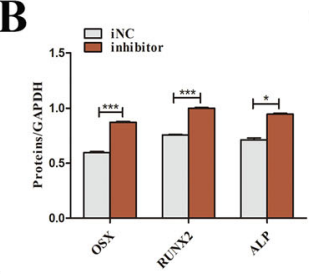

C
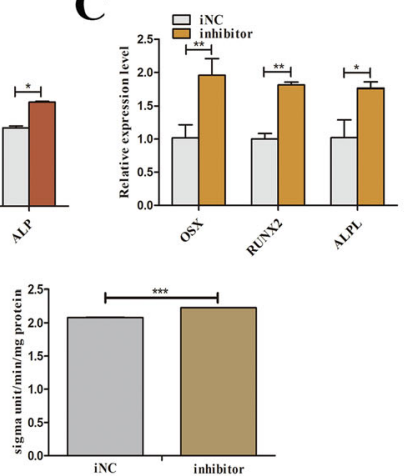

ys
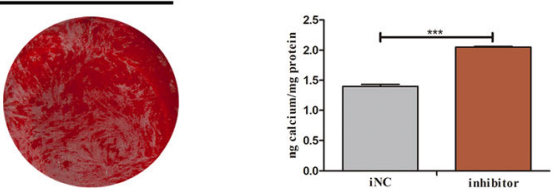

inhibitor

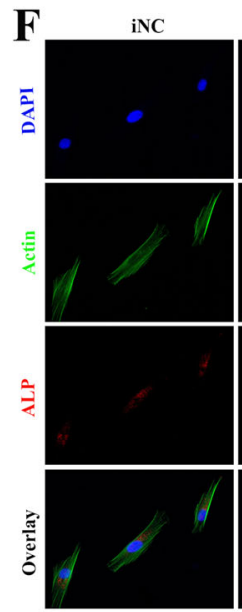

inhibitor

iNC
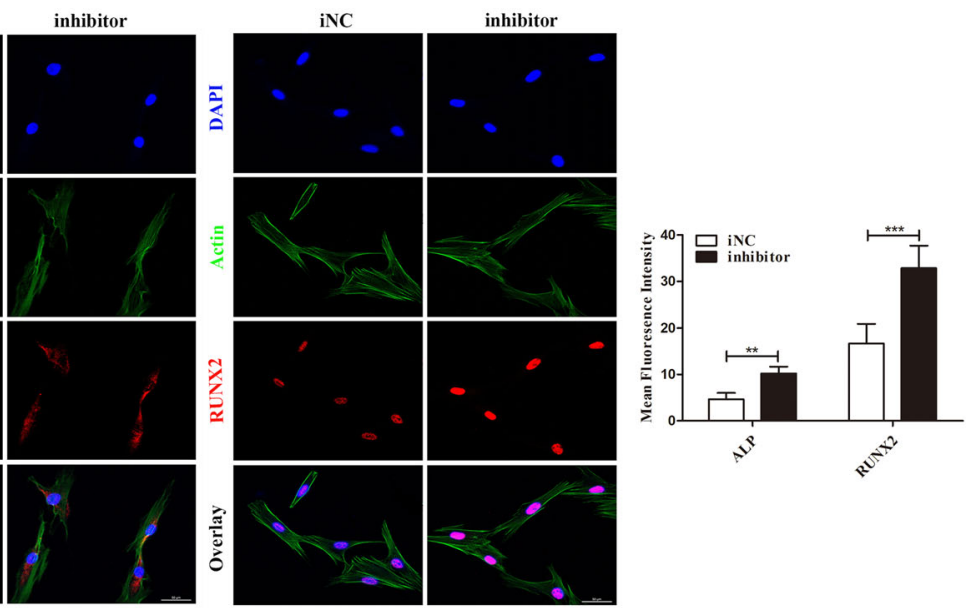

Fig. 5 Knockdown of miR-204-5p enhanced osteogenic differentiation of SCAPs. a Western blot assay showed lower protein levels of ALP, RUNX2, and OSX in the inhibitor-NC group than the miR-204-5p inhibitor group. GAPDH was the internal control. b Grayscale analyses. c qRT-PCR showed higher levels of ALPL, RUNX2, and OSX in the miR-204-5p inhibitor group. $\mathbf{d}$ ALP staining and ALP activity assay were performed to evaluate the expression of ALP in the inhibitor-NC and miR-204-5p inhibitor group at day 5. e Alizarin red staining showed that the miR-204-5p inhibitor group generated more calcified nodules than the control group on day 14. CPC assay showed quantification of Alizarin red staining by spectrophotometry. f Immunofluorescence staining showed upregulated ALP and RUNX2 in the miR-204-5p inhibitor group compared with the NC group (scale bar $=50 \mu \mathrm{m}$ ). Quantification was done by ImageJ. ${ }^{*} P<0.05$, ${ }^{* *} P<0.01$, and ${ }^{* * *} P<0.001$ 
mineralization nodule formation compared with SCAPs transfected with mimic-NC group after 5 and 14 days, respectively (Fig. $4 \mathrm{~d}$, e). ALP activity assay and $\mathrm{CPC}$ results further confirmed these effects $(P<$ 0.01 , Fig. $4 g, h)$. Similar to the results of Western blot analysis, we observed a consistent reduction in the protein levels of ALP and RUNX2 in miR-204-5p group by immunofluorescence assay (Fig. 4i).

\section{Knockdown of miR-204-5p promotes osteogenic differentiation of SCAPs}

SCAPs treated with miR-204-5p inhibitor and inhibitor negative control was induced to differentiate for 3 days. Both mRNA and protein levels of ALP, RUNX2, and OSX were significantly upregulated in miR-204-5p inhibitor group $(P<0.05$ or $P<0.01$, Fig. 5a-c). In addition, ALP staining and Alizarin red staining showed that miR-204-5p inhibitor promotes expression of ALP and formation of mineralized nodules of SCAPs (Fig. 5d, e). Immunofluorescence assay further illustrated that the protein levels of ALP and RUNX2 were upregulated in the miR-204-5p inhibitor group (Fig. 5f).

\section{Bioinformatic analysis of miR-204-5p target gene}

To predict potential target genes of miR-204-5p, bioinformatics analysis was performed by TargetScan, miRWalk, miRTarBase, and MiRDB. 11,310 potential target genes were acquired, of which 113 were in common in the above 4 databases (Fig. 6a). Furthermore, GO annotation and KEGG pathway analysis showed that these target genes are involved in a multiple biological process and pathways, such as the MAPK signaling pathway that has been widely studied (Fig. 6b, c). Interestingly, ALPL as a critical gene in osteogenesis was one of the common target genes of miR-204-5p.

\section{CircSIPA1L1 affects the expression of ALPL by serving as a miRNA sponge for miR-204-5p}

To identify the interaction of circSIPA1L1 and miR-204$5 \mathrm{p}$, we simultaneously performed nuclear/cytoplasmic separation and FISH experiments. After separating cytoplasmic and nuclear fractions, the expression ratio of circSIPA1L1 was detected by qPCR. Almost $90 \%$ of circSIPA1L1 transcripts were measured in the cytoplasm (Fig. 7d). Furthermore, the results of FISH showed that circSIPA1L1 and miR-204-5p is mainly exist in the cytoplasm (Fig. 7e, f). These results suggested that circSIPA1L1 may have potential biological roles in posttranscriptional regulation.

To understand the underlying mechanism that circSIPA1L1 regulates osteogenic differentiation of SCAPs, we tested the expression of circSIPA1L1 after the transfection of miR-204-5p. Notably, we observed a negative correlation between circSIPA1L1 and miR204-5p $(P<0.05$ or $P<0.01$, Fig. 7c). To further explore the relationship between circSIPA1L1 and miR204-5p, the luciferase reporter plasmid was constructed by cloning the putative miR-204-5p target binding sequence of circSIPA1L1 into a luciferase plasmid vector. MiR-204-5p mimics and circSIPA1L1 wild-type or mutant luciferase plasmid were transferred into $293 \mathrm{~T}$ cells. MiR-204-5p mimics significantly suppressed the luciferase activity of circSIPA1L1 wild-type reporter, but this phenomenon was successfully reversed by mutation of the putative miR-204-5p target sites $(P<0.01$, Fig. 7a). Similarly, we observed a consistent reduction of luciferase activity in $293 \mathrm{~T}$ co-transfected with miR-204-5p and
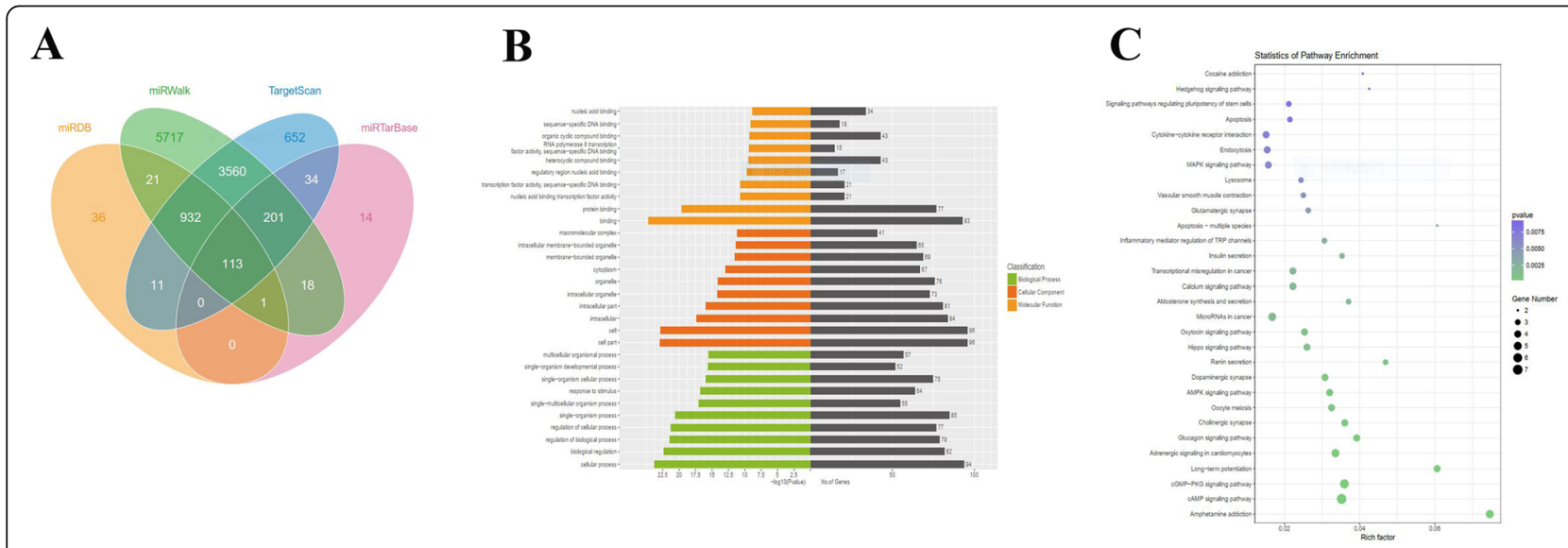

Fig. 6 Bioinformatic analysis on the target gene of miR-204-5p. a Venn diagram showed the number of miR-204-5p target genes predicted by performing miRDB, miRTarBase, miRWalk, and TargetScan algorithms. b, c GO annotation (b) and KEGG pathway analysis (c) showed the top 30 target genes and their enriched pathways. GO: domains directly related with reproduction 
A
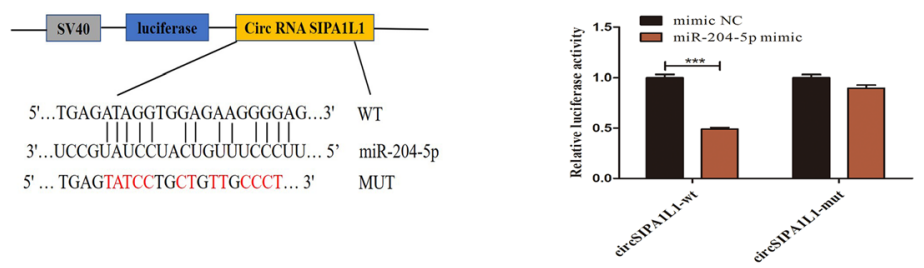

B
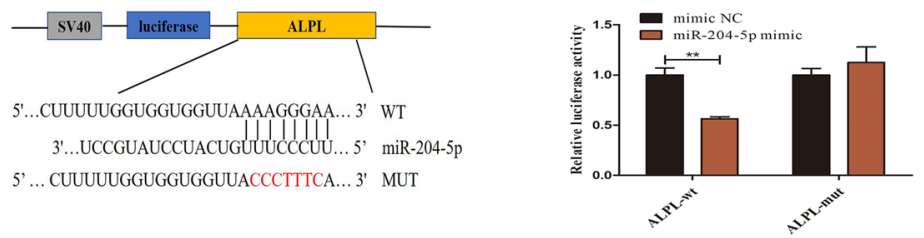

C
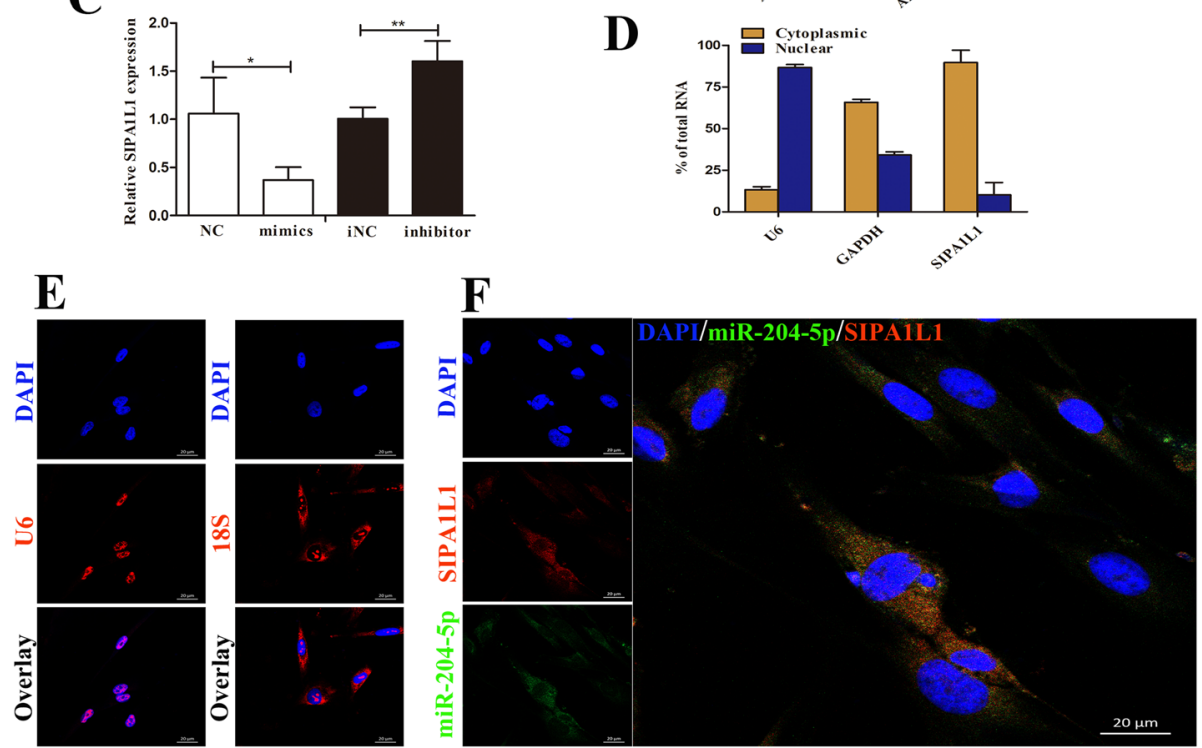

Fig. 7 CircSIPA1L1 affects the expression of ALPL by serving as a miRNA sponge for miR-204-5p. a Schematic diagram of miR-204-5p with the seed sequences of wild-type circSIPA1L1 3'UTR (WT) and mutant circSIPA1L1 3'UTR (Mut). Luciferase reporter assays in 293 T illustrated that miR-204-5p could bind with circSIPA1L1. b The mutant ALPL-3'-UTR reporter plasmid was constructed. Luciferase reporter assays in 293 T showed that miR-204-5p could bind with ALPL. c Relative circSIPA1L1 expression level in SCAPs transfected with miR-204-5p mimics or inhibitor. $\mathbf{d}$ Cytoplasmic and nuclear fractions of cellular RNA were analyzed by qRT-PCR and expressed as a percentage of the input. U6 and GAPDH mRNA were used as reference RNAs for nuclear and cytoplasmic fractions, respectively. e, f Co-localization between miR-204-5p and circSIPA1L1 was observed by FISH in SCAPs (f). 185 and U6 were the internal control (e). Scale bar $=50 \mu \mathrm{m} .{ }^{*} P<0.05,{ }^{* *} P<0.01$

ALPL wild-type $(P<0.01$, Fig. $7 \mathrm{~b})$. Furthermore, the expression of ALPL was correspondingly negatively regulated by miR-204-5p $(P<0.001$, Fig. 4c). Altogether, these results provided that circSIPA1L1 can affect the expression of ALPL by targeting miR204-5p.

\section{CircSIPA1L1/miR-204-5p/ALPL regulates osteogenic differentiation of SCAPs}

To further identify the miR-204-5p that binds to circSIPA1L1 regulating ALPL expression to promote osteoblast differentiation of SCAPs, the rescue experiments were performed. SCAPs were treated with siSIPA1L1, miR-204-5p inhibitor, and the corresponding si-NC, inhibitor-NC. qRT-PCR and Western blot results suggested that co-transfection with si-SIPA1L1 and miR-204-5p inhibitor partially blocked the mRNA and protein expression levels of ALPL/ALP, RUNX2/ RUNX2, and $O S X / O S X$, in comparison to the siSIPA1L1 group $(P<0.05$ or $P<0.01$, Fig. $8 \mathrm{a}, \mathrm{b})$. As expected, ALP staining and ALP activity result further confirmed this phenomenon (Fig. 8c, d). Taken together, circSIPA1L1 absorbs miR-204-5p as a competitive endogenous RNA, increases the transcription of ALPL to promote the osteogenic differentiation of SCAPs (Fig. 8e).

\section{Discussion}

It is well known that tissue engineering based on SCAPs has been widely reported, but the functional significance of 

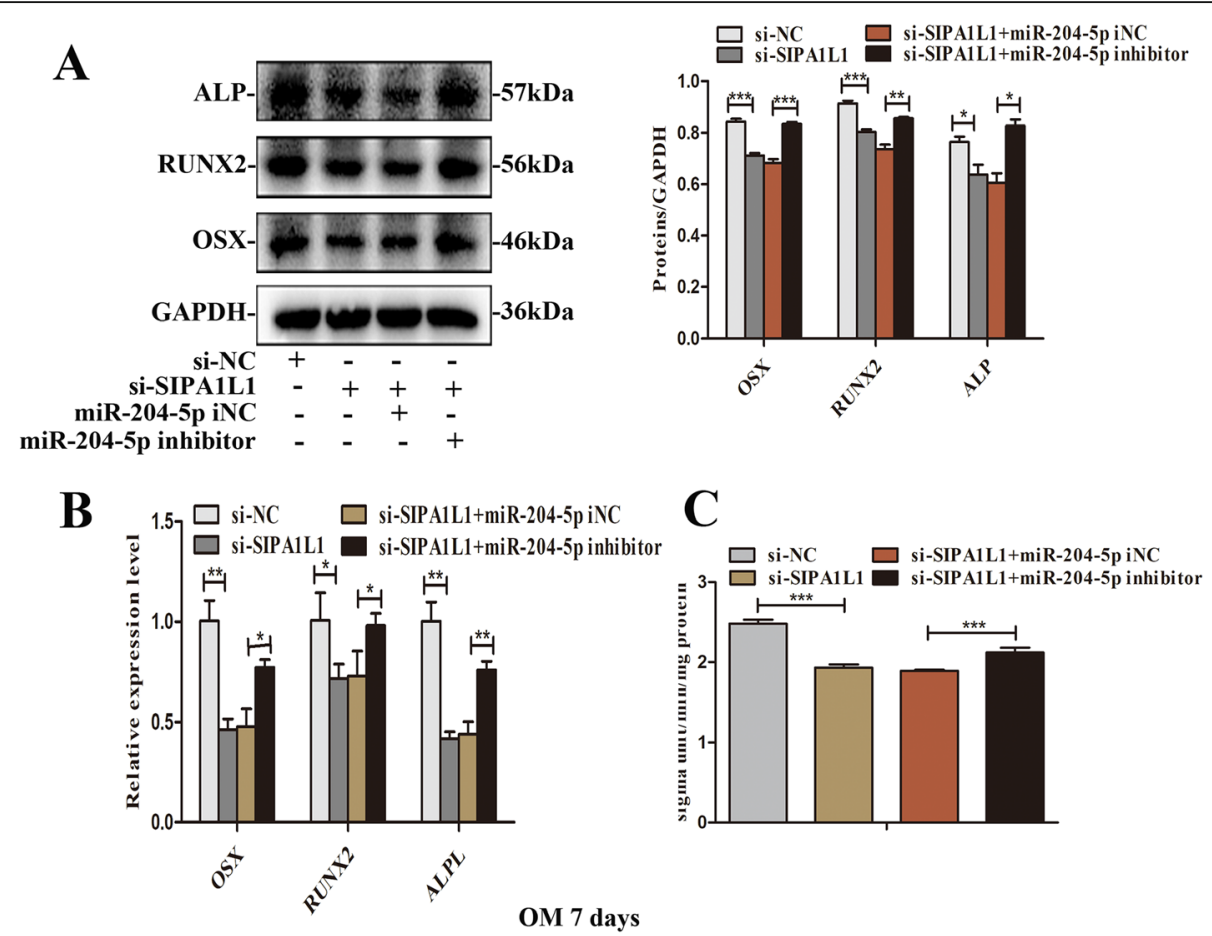

D
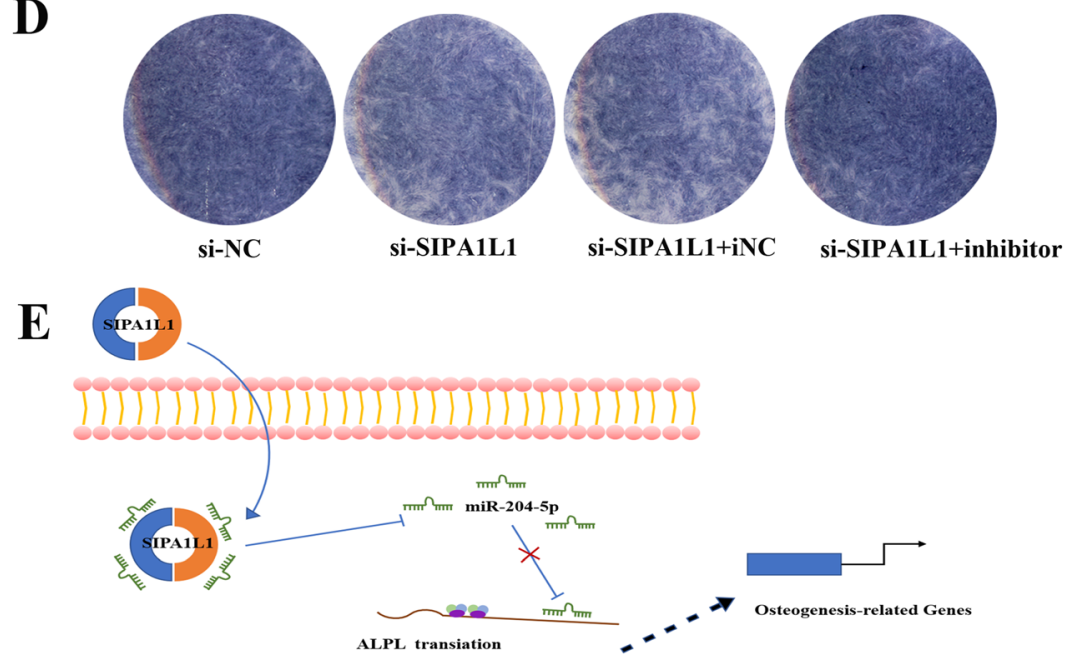

Fig. 8 CircSIPA1L1/miR-204-5p/ALPL regulates osteogenic differentiation of SCAPs. a Results of western blot analysis indicated that the miR-204$5 p$ inhibitor rescued the si-SIPA1L1 mediated downregulation of ALP, RUNX2, and OSX. GAPDH served as an internal control. Histograms showed the quantification of band intensities. $\mathbf{b}$ Results of qRT-PCR analysis revealed the miR-204-5p inhibitor rescued the si-SIPA1L1 mediated downregulation of ALPL, RUNX2, and OSX. $\mathbf{c}$, d ALP staining and ALP activity assay showed the miR-204-5P inhibitor rescued the si-SIPA1L1 mediated downregulation expression of ALP. e Schematic diagram for circSIPA1L1/ miR-204-5p /ALPL axis. ${ }^{*} P<0.05$, ${ }^{*} P<0.01$, and ${ }^{* *} P<0.001$

noncoding RNA in SCAPs in tissue regeneration remains largely unknown. In our previous research, a large number of circRNAs were detected in SCAPs [31]. More importantly, we found that the expression of circSIPA1L1 was down expression while miR-204-5p was upregulated in SCAPs during the osteogenic differentiation, suggesting that circSIPA1L1 and miR-204-5p may involve in the osteogenic differentiation of SCAPs. In our study, qRT-PCR and Western blot analysis indicated that overexpression circSIPA1L1 promotes osteogenic differentiation of SCAPs in vitro. Meanwhile, ALP and ARS staining also confirmed this conclusion. Similarly, knocking down circSIPA1L1 further showed that circSIPA1L1 stimulated osteogenic differentiation. In addition, we did not observe the effect of circSIPA1L1 on cell proliferation of SCAPs. In view of the complexity of cell proliferation, whether circSIPA1L1 at different transfection concentrations or different cell lineages have different effects on proliferation may require further investigation. 
We found that circSIPA1L1 and miR-204-5p were prominently co-localized in the cytoplasm of SCAPs by the FISH (Fig. 6e, f). Hence, we hypothesized that circSIPA1L1 could have functional significances in the cytoplasm. However, the function of circRNAs in the cytoplasm is diverse. Some circRNAs works in the cytoplasm to form circRNPs (circRNA-protein complexes) by adsorbing proteins [37]. For example, circFoxo3 interacted with the anti-stress proteins FAK and HIF1 $\alpha$, as well as the anti-senescent protein ID-1 and the transcription factor E2F1, leading to promoted cell aging [38]. In addition, circZNF609 can also actively translate to exert protein-coding ability in myoblasts, in view of the association with heavy polysomes [39]. Although almost all circular RNAs are exclusively present in the cytoplasm, there is still a small portion of circRNAs are restricted in the nucleus and bind to small nuclear ribonucleoproteins to regulate the transcription of certain genes [40].

Growing evidence identified that circRNAs are mainly located in the cytoplasm, where it affects gene expression through the action of the microRNA sponge [41]. In this research, the luciferase activity of circSIPA1L1 wild-type and miR-204-5p wild-type were remarkably decreased by miR-204-5p mimics (Fig. 6a, b). The expression of bonerelated genes and proteins including ALPL/ALP were downregulated in the si-SIPA1L1 group, while cotransfection with miR-204-5p inhibitor and si-SIPA1L1 partially reversed this effect (Fig. 8a, b). These results indicated that circSIPA1L1 regulates ALPL expression through the classical model of sponging miR-204-5p. Interestingly, the miR-204-5p inhibitor did not completely block the effects of circSIPA1L1 knockdown, suggesting the role of circSIPA1L1 on the regulation of osteogenic differentiation could be complicated. Whether there are other regulatory mechanisms of circSIPA1L1 is worth further exploration.

According to reports, Ingenuity Pathway Analysis results showed that miR-204-5p can directly target ALPL [28]. Here, the bioinformatics analysis and luciferase reporter experiments further proved that miR-204-5p targets the 3'UTR of the ALPL gene, inhibiting translation of ALPL at the post-transcriptional level. ALPL gene is also essential for the normal development and homeostasis of the bones and teeth, because it encodes ALP. As we all know, ALP promotes mineralization by hydrolyzing pyrophosphate to release inorganic phosphate which binds to calcium [42]. The low circulating concentration of total ALP is a characteristic feature of a rare genetic disease called hypophosphatasia that causes osteoporosis, osteomalacia, fragility fractures, and dental problems in adults [43]. The cause of hypophosphatasia is loss-of-function or dominant-negative mutations in ALPL. Heterozygous ALPL mutations can lead to low serum ALP levels.
Persistent low serum ALP may be related to non-specific musculoskeletal symptoms [44]. A genetic study suggested that ALPL is necessary for postnatal bone formation. The study also further clarified that the bone deformities are related to the degree of ALPL deficiency [30]. Taken together, these results revealed that ALPL plays a critical role in bone formation. Our results show that circSIPA1L1 can significantly promote the expression of ALP, which may provide some reference for bone regeneration and even provide a new insight for the etiology and treatment of hypophosphatasia.

\section{Conclusion}

In conclusion, our results elucidated the osteogenic function of circSIPA1L1 in SCAPs. Our findings suggest that circSIPA1L1 serves as an endogenous miR-204-5p sponge to promote osteogenic differentiation of SCAPs, which may be used as a potential target for revealing the molecular mechanism of the osteogenic differentiation of SCAPs.

\section{Abbreviations}

CircSIPA1L1: Circular RNA SIPA1L1; SCAPs: Stem cells from apical papilla; ALP: Alkaline phosphatase; ARS: Alizarin red staining; MSCs: Mesenchymal stem cells; miRNA: MicroRNA; ceRNAs: Competitive endogenous RNAs; NC: Negative control; RT-PCR: Reverse transcription polymerase chain reaction; CPC: Cetylpyridinium chloride; FISH: Fluorescence in situ hybridization

\section{Acknowledgements}

Not applicable.

\section{Authors' contributions}

Yuzhi Li, Minxia Bian, and Zhou Zhou performed study design, data collection, and wrote the manuscript. Xiao Wu and Xingyun Ge completed data analysis and interpretation. Tong Xiao reviewed the data. Jinhua Yu conceived and designed the study, provided financial support and study material, performed data analysis and interpretation, and approved the final version of the manuscript. The authors read and approved the manuscript.

\section{Funding}

This work was supported by the National Natural Science Foundation of China (grant numbers: 81873707 and 81900962), Medical Talent Project of Jiangsu Province (grant number: ZDRCA2016086), the Priority Academic Program Development of Jiangsu Higher Education Institutions (PAPD, grant number: 2018-87), and Science and Technology Development Project of Jiangsu Province (grant number: BE2017731).

\section{Availability of data and materials}

The datasets used and analyzed during the current study are available from the corresponding author on reasonable request.

Ethics approval and consent to participate

Studies were carried out in accordance with the Declaration of Helsinki and got the approval of the Ethical Committee of Nanjing Medical University.

\section{Consent for publication \\ Not applicable.}

Competing interests

The authors declare that they have no competing interests. 
Received: 10 September 2020 Accepted: 11 October 2020 Published online: 02 November 2020

\section{References}

1. Wei F, Yang S, Guo Q, Zhang X, Ren D, et al. MicroRNA-21 regulates osteogenic differentiation of periodontal ligament stem cells by targeting Smad5. Sci Rep. 2017;7:16608. https://doi.org/10.1038/s41598-017-16720-8.

2. Cherubini A, Barilani M, Rossi RL, Jalal MMK, Rusconi F, et al. FOXP1 circular RNA sustains mesenchymal stem cell identity via microRNA inhibition. Nucleic Acids Res. 2019;47:5325-40. https://doi.org/10.1093/nar/gkz199.

3. Sonoyama W, Liu Y, Yamaza T, Tuan RS, Wang S, et al. Characterization of the apical papilla and its residing stem cells from human immature permanent teeth: a pilot study. J Endodont. 2008;34:166-71. https://doi.org/ 10.1016/j.joen.2007.11.021.

4. Wang $Y$, Pang $X, W u J$, Jin $L, Y u Y$, et al. MicroRNA hsa-let-7b suppresses the odonto/osteogenic differentiation capacity of stem cells from apical papilla by targeting MMP1. J Cell Biochem. 2018;119:6545-54. https://doi.org/10. 1002/jcb.26737

5. Yang C, Li XH, Sun L, Guo WH, Tian WD. Potential of human dental stem cells in repairing the complete transection of rat spinal cord. J Neural Eng. 2017;14:ARTN 026005. https://doi.org/10.1088/1741-2552/aa596b.

6. Deng J, Pan J, Han X, Yu L, Chen J, et al. PDGFBB-modified stem cells from apical papilla and thermosensitive hydrogel scaffolds induced bone regeneration. Chem Biol Interact. 2020;316:108931. https://doi.org/10.1016/j. cbi.2019.108931

7. Devillard R, Rémy M, Kalisky J, Bourget JM, Kérourédan O, et al. In vitro assessment of a collagen/alginate composite scaffold for regenerative endodontics. Int Endod J. 2017:50:48-57. https://doi.org/10.1111/iej.12591.

8. Saberi E, Farhad-Mollashahi N, Sargolzaei Aval F, Saberi M. Proliferation, odontogenic/osteogenic differentiation, and cytokine production by human stem cells of the apical papilla induced by biomaterials: a comparative study. Clin Cosmet Investig Dent. 2019;11:181-93. https://doi.org/10.2147/ CCIDE.S211893.

9. Jarroux J, Morillon A, Pinskaya M. History, discovery, and classification of IncRNAs. Adva Exp Med Biol. 2017;1008:1-46. https://doi.org/10.1007/978981-10-5203-3_1.

10. Gao Y, Wang JF, Zhao FQ. CIRI: an efficient and unbiased algorithm for de novo circular RNA identification. Genome Biol. 2015;16:ARTN 4. https://doi. org/10.1186/s13059-014-0571-3.

11. Starke S, Jost I, Rossbach O, Schneider T, Schreiner S, et al. Exon circularization requires canonical splice signals. Cell Rep. 2015:10:103-11. https://doi.org/10.1016/j.celrep.2014.12.002.

12. Huang S, Li X, Zheng H, Si X, Li B, et al. Loss of super-enhancer-regulated circRNA Nfix induces cardiac regeneration after myocardial infarction in adult mice. Circulation. 2019;139:2857-76. https://doi.org/10.1161/ circulationaha.118.038361.

13. Li R, Jiang J, Shi H, Qian H, Zhang $X$, et al. CircRNA: a rising star in gastric cancer. Cell Mol Life Sci. 2020;77:1661-80. https://doi.org/10.1007/s00018019-03345-5.

14. Kristensen LS, Andersen MS, Stagsted LWW, Ebbesen KK, Hansen TB, et al. The biogenesis, biology and characterization of circular RNAs. Nat Rev Genet. 2019;20:675-91. https://doi.org/10.1038/s41576-019-0158-7.

15. Cooper DA, Cortés-López M, Miura P. Genome-wide circRNA profiling from RNA-seq data. Methods Mol Biol (Clifton, N.J.). 2018;1724:27-41. https://doi. org/10.1007/978-1-4939-7562-4_3.

16. Yang M, Xiang G, Yu D, Yang G, He W, et al. Hsa circ 0002468 regulates the neuronal differentiation of SH-SY5Y cells by modulating the MiR-561/E2F8 axis. Med Sci Monit. 2019;25:2511-9. https://doi.org/10.12659/msm.915518.

17. Han J, Zhao G, Ma X, Dong Q, Zhang H, et al. CircRNA circ-BANP-mediated miR-503/LARP1 signaling contributes to lung cancer progression. Biochem Biophys Res Commun. 2018;503:2429-35. https://doi.org/10.1016/j.bbrc.2018. 06.172 .

18. Peng S, Song C, Li H, Cao X, Ma Y, et al. Circular RNA SNX29 sponges miR744 to regulate proliferation and differentiation of myoblasts by activating the Wnt5a/Ca(2+) signaling pathway. Mol Ther Nucleic Acids. 2019;16:48193. https://doi.org/10.1016/j.omtn.2019.03.009.

19. Li $X$, Zheng $Y$, Zheng $Y$, Huang $Y$, Zhang $Y$, et al. Circular RNA CDR1as regulates osteoblastic differentiation of periodontal ligament stem cells via the miR-7/GDF5/SMAD and p38 MAPK signaling pathway. Stem Cell Res Ther. 2018;9:232. https://doi.org/10.1186/s13287-018-0976-0.
20. Ouyang Z, Tan T, Zhang X, Wan J, Zhou Y, et al. CircRNA hsa_circ_0074834 promotes the osteogenesis-angiogenesis coupling process in bone mesenchymal stem cells (BMSCs) by acting as a ceRNA for miR-942-5p. Cell Death Dis. 2019;10:932. https://doi.org/10.1038/s41419-019-2161-5.

21. Lu TX, Rothenberg ME. MicroRNA. J Allergy Clin Immunol. 2018;141:1202-7. https://doi.org/10.1016/j.jaci.2017.08.034.

22. Han X, Yang H, Cao Y, Ge L, Han N, et al. The miR-3940-5p inhibits cell proliferation of gingival mesenchymal stem cells. Oral Dis. 2019;25:1363-73. https://doi.org/10.1111/odi.13092.

23. Li J, Hu C, Han L, Liu L, Jing W, et al. MiR-154-5p regulates osteogenic differentiation of adipose-derived mesenchymal stem cells under tensile stress through the Wnt/PCP pathway by targeting Wnt11. Bone. 2015;78:130-41. https://doi.org/10.1016/j. bone.2015.05.003.

24. Palkina N, Komina A, Aksenenko M, Moshev A, Savchenko A, et al. miR-204$5 p$ and miR-3065-5p exert antitumor effects on melanoma cells. Oncol Lett. 2018;15:8269-80. https://doi.org/10.3892/ol.2018.8443.

25. Hong BS, Ryu HS, Kim N, Kim J, Lee E, et al. Tumor suppressor miRNA-204$5 p$ regulates growth, metastasis, and immune microenvironment remodeling in breast cancer. Cancer Res. 2019;79:1520-34. https://doi.org/ 10.1158/0008-5472.CAN-18-0891.

26. He H, Chen K, Wang F, Zhao L, Wan X, et al. miR-204-5p promotes the adipogenic differentiation of human adipose-derived mesenchymal stem cells by modulating DVL3 expression and suppressing Wnt/beta-catenin signaling. Int J Mol Med. 2015;35:1587-95. https://doi.org/10.3892/ijmm.2015.2160.

27. Yu C, Li L, Xie F, Guo S, Liu F, et al. LncRNA TUG1 sponges miR-204-5p to promote osteoblast differentiation through upregulating Runx2 in aortic valve calcification. Cardiovasc Res. 2018;114:168-79. https://doi.org/10.1093/ $\mathrm{cvr} / \mathrm{cv} \times 180$

28. Liu F, Wang $X$, Yang $Y$, Hu R, Wang W, et al. The suppressive effects of miR508-5p on the odontogenic differentiation of human dental pulp stem cells by targeting glycoprotein non-metastatic melanomal protein B. Stem Cell Res Ther. 2019;10:35. https://doi.org/10.1186/s13287-019-1146-8.

29. Alonso N, Larraz-Prieto B, Berg K, Lambert Z, Redmond P, et al. Loss-offunction mutations in the ALPL gene presenting with adult onset osteoporosis and low serum concentrations of total alkaline phosphatase. J Bone Miner Res. 2019. https://doi.org/10.1002/jbmr.3928.

30. Liu W, Zhang L, Xuan K, Hu C, Liu S, et al. Alpl prevents bone ageing sensitivity by specifically regulating senescence and differentiation in mesenchymal stem cells. Bone Res. 2018;6:27. https://doi.org/10.1038/ s41413-018-0029-4.

31. Li Z, Li N, Ge X, Pan Y, Lu J, et al. Differential circular RNA expression profiling during osteogenic differentiation of stem cells from apical papilla. Epigenomics. 2019;11:1057-73. https://doi.org/10.2217/epi-2018-0184.

32. Ge X, Li Z, Zhou Z, Xia Y, Bian M, et al. Circular RNA SIPA1L1 promotes osteogenesis via regulating the miR-617/Smad3 axis in dental pulp stem cells. Stem Cell Res Ther. 2020;11:364. https://doi.org/10.1186/s13287-020-01877-3.

33. Li Z, Ge X, Lu J, Bian M, Li N, et al. MiR-141-3p regulates proliferation and senescence of stem cells from apical papilla by targeting YAP. Exp Cell Res. 2019;383:111562. https://doi.org/10.1016/j.yexcr.2019.111562.

34. Liu GX, Ma S, Li Y, Yu Y, Zhou YX, et al. Hsa-let-7c controls the committed differentiation of IGF-1-treated mesenchymal stem cells derived from dental pulps by targeting IGF-1R via the MAPK pathways. Exp Mol Med. 2018;50:25 https://doi.org/10.1038/s12276-018-0048-7.

35. Ma $S$, Liu G, Jin L, Pang $X$, Wang $Y$, et al. IGF-1/IGF-1R/hsa-let-7c axis regulates the committed differentiation of stem cells from apical papilla. Sci Rep. 2016;6:36922. https://doi.org/10.1038/srep36922.

36. Wu J, Li N, Fan Y, Wang Y, Gu Y, et al. The conditioned medium of calcined tooth powder promotes the osteogenic and odontogenic differentiation of human dental pulp stem cells via MAPK signaling pathways. Stem Cells Int. 2019;2019:4793518. https://doi.org/10.1155/2019/4793518.

37. Schneider T, Hung LH, Schreiner S, Starke S, Eckhof H, et al. CircRNA-protein complexes: IMP3 protein component defines subfamily of circRNPs. Sci Rep. 2016;6:31313. https://doi.org/10.1038/srep31313.

38. Du WW, Yang W, Chen Y, Wu ZK, Foster FS, et al. Foxo3 circular RNA promotes cardiac senescence by modulating multiple factors associated with stress and senescence responses. Eur Heart J. 2017;38:1402-12. https:// doi.org/10.1093/eurheartj/ehw001.

39. Legnini I, Di Timoteo G, Rossi F, Morlando M, Briganti F, et al. Circ-ZNF609 is a circular RNA that can be translated and functions in myogenesis. Mol Cell. 2017;66:22-37.e9. https://doi.org/10.1016/j.molcel.2017.02.017. 
40. Li X, Yang L, Chen LL. The biogenesis, functions, and challenges of circular RNAs. Mol Cell. 2018;71:428-42. https://doi.org/10.1016/j.molcel.2018.06.034.

41. Qu S, Yang X, Li X, Wang J, Gao Y, et al. Circular RNA: a new star of noncoding RNAs. Cancer Lett. 2015;365:141-8. https://doi.org/10.1016/j. canlet.2015.06.003

42. Misawa A, Orimo H. IncRNA HOTAIR inhibits mineralization in osteoblastic osteosarcoma cells by epigenetically repressing ALPL. Calcif Tissue Int. 2018; 103:422-30. https://doi.org/10.1007/s00223-018-0434-0.

43. Whyte MP. Hypophosphatasia: an overview for 2017. Bone. 2017;102:15-25. https://doi.org/10.1016/j.bone.2017.02.011.

44. Rauch F, Bardai G, Rockman-Greenberg C. ALPL mutations in adults with rheumatologic disorders and low serum alkaline phosphatase activity. J Bone Miner Metab. 2019;37:893-9. https://doi.org/10.1007/s00774-01900991-4.

\section{Publisher's Note}

Springer Nature remains neutral with regard to jurisdictional claims in published maps and institutional affiliations.

Ready to submit your research? Choose BMC and benefit from:

- fast, convenient online submission

- thorough peer review by experienced researchers in your field

- rapid publication on acceptance

- support for research data, including large and complex data types

- gold Open Access which fosters wider collaboration and increased citations

- maximum visibility for your research: over $100 \mathrm{M}$ website views per year

At BMC, research is always in progress.

Learn more biomedcentral.com/submissions 